\title{
Miteinander statt nebeneinander! Strategien für die Weiterentwicklung interkommunaler Kooperationsstrukturen in der „Wiener Stadtregion ${ }^{+6}$
}

\section{Together, not alongside! Strategies for the development of inter-municipal cooperation in the Stadtregion ${ }^{+}$of Vienna}

https://doi.org/10.2478/rara-2020-0018

Eingegangen: 3. Dezember 2019; Angenommen: 12. Mai 2020

Kurzfassung: Die Wiener Stadtregion+ zählt zu den dynamischsten Wachstumsregionen Europas. Das Management des Bevölkerungswachstums stellt Stadt und Umland vor umfangreiche planungspolitische Herausforderungen, die eine gemeinsame und abgestimmte Vorgehensweise erforderlich machen. Die diesbezüglichen stadtregionalen Kooperationsstrukturen sind gegenwärtig inhaltlich und institutionell jedoch nur schwach ausgeprägt. Ziel des Beitrags ist es, vor diesem Hintergrund auf analytischer und instrumenteller Ebene Vorschläge zur Weiterentwicklung von informell-kooperativen Raumentwicklungsprozessen in Stadtregionen auszuarbeiten. Die theoretische Einbettung erfolgt über das Konzept der strategischen Planung in Kombination mit dem Ansatz der soft spaces. Anhand des „Verflechtungs-Kooperations-Paradoxons" werden dabei auf analytischer Ebene die sehr unterschiedlichen Strukturen funktionaler Verflechtungen und interkommunaler Kooperationen in der Stadtregion ${ }^{+}$aufgezeigt. Auf instrumenteller Ebene wird das Planungsprinzip der „flexiblen Regionalisierungen“ vorgestellt, das die Idee räumlicher und zeitlicher Flexibilität in der strategischen Planung aufgreift und weiterdenkt. Ein wesentlicher Bestandteil davon ist die Identifizierung von „Fokusräumen“ als spezifische Teilbereiche innerhalb einer Stadtregion. Dabei handelt es sich um ,weiche Planungsräume', in denen interkommunal, kooperativ und umsetzungsorientiert an gemeinsamen Planungs- und Entwicklungsherausforderungen gearbeitet wird. Die gewählte Methodik sowie die daraus resultierenden Ergebnisse werden kritisch reflektiert und diskutiert, bevor abschließend wienspezifische und allgemeine Handlungsempfehlungen abgeleitet werden.

Schlüsselwörter: Stadtregion Wien, Kooperation, soft spaces, funktionale Verflechtung, Fokusraum, strategische Planung

\footnotetext{
*Corresponding author: Stefanie Döringer, Universität Wien, Institut für Geographie und Regionalforschung, Universitätsstraße 7 , 1010 Wien, Österreich; Österreichische Akademie der Wissenschaft, Institut für Stadt- und Regionalforschung, Postgasse 7/4/2, 1010 Wien, Österreich, E-Mail: stefanie.doeringer@oeaw.ac.at, ORCID: 0000-0002-7817-4698 Dr. Peter Görgl, Universität Wien, Institut für Geographie und Regionalforschung, Universitätsstraße 7, 1010 Wien, Österreich, ORCID: 0000-0003-3254-2149

Johannes Herburger, Universität Liechtenstein, Institut für Architektur und Raumentwicklung, Fürst-Franz-Josef-Straße, 9490 Vaduz, Liechtenstein, ORCID: 0000-0003-4792-182X
} 
Abstract: The urban region of Vienna (Stadtregion ${ }^{+}$) is among the most dynamic growth regions in Europe. The management of the population growth poses extensive challenges for the city and its hinterland requiring a common and coordinated procedure. The urban regional cooperation structures are currently weakly institutionalised and barely defined. Against this background, this paper aims at presenting recommendations for the development of informal-cooperative spatial development processes in city regions on an analytical and instrumental level. The theoretical framework is based on a combination of the concept of strategic planning and the approach of soft spaces. The methods include the analysis of functional interconnections, a network analysis as well as the calculation of an interconnection index. By means of the "paradox of interconnection and cooperation" the totally different structures of the city-regional functional interconnections and the inter-municipal cooperation are shown on an analytical level. On an instrumental level, the planning principle of "flexible regionalisation" is presented, which draws upon and further develops the idea of spatial and temporal flexibility in strategic planning. A central element of this principle is the identification of "urban-regional focus areas" defined as specific areas within the urban region. These focus areas are characterised as soft planning spaces, in which to work on common planning and development challenges in an inter-municipal, cooperative and implementation-oriented manner. The applied methods and its findings are critically reflected and discussed, before deriving Vienna-specific and general policy recommendations.

Keywords: Urban region of Vienna, Cooperation, Soft Spaces, Functional Interconnections, Focus Area, Strategic Planning

\section{Einleitung}

Die österreichische Bundeshauptstadt Wien und ihr Verflechtungsraum zählen seit gut zehn Jahren zu den europäischen Stadtregionen, die sich am dynamischsten entwickeln (Stadt Wien 2014b: 13; Hanika/Fuchs/Klotz 2015: 133 f.). Entsprechend komplex sind die damit verbundenen planungs- und entwicklungspolitischen Herausforderungen. Stadtregionales Wachstumsmanagement muss sich nicht nur um den quantitativen Anstieg von Bevölkerung, Verkehr oder Gewerbe kümmern, sondern auch um konkurrierende Raumansprüche, die aufgrund von nurmehr begrenzt vorhandenen Flächenpotenzialen stetig zunehmen.

Während sich die Gemeinden im Wiener Umland zunehmend mit Planungsherausforderungen von stadtregionaler Relevanz konfrontiert sehen, sind in der österreichischen Bundeshauptstadt die für die weitere Entwicklung notwendigen Flächen knapp. Aus fachlicher Sicht ist daher eine entsprechend intensive stadtregionale Kooperation notwendig, um diese komplexe Gemengelage in der sogenannten Stadtregion ${ }^{+}$planerisch und politisch bewältigen zu können. Bis heute existieren Stadt-Umland-Kooperationen allerdings nur auf einem wenig institutionalisierten und weitestgehend informellen Niveau (Görgl/Gruber 2015: 272 ff.). Ebenso gibt es keine gemeinsam formulierte Planungs- und Entwicklungsstrategie, die auf fachlicher oder politischer Ebene strategisch-steuernde Wirkung hätte. Der Kooperations- und Steuerungsbedarf der stadtregionalen Ent- wicklung wird von politischen Mandataren und vonseiten der involvierten Fachabteilungen immer wieder betont. So wird beispielsweise im Auftrag der Bundesländer Wien, Niederösterreich und Burgenland kontinuierlich zu diesen Themen geforscht und ein fachlicher Austausch in länderübergreifenden Institutionen gepflegt (Fassmann/ Görgl/Helbich 2009; Döringer/Görgl/Huemer 2014; Eder/ Gruber/Görgl et al. 2018). Es liegt also nahe, ganz grundsätzlich danach zu fragen, wie die derzeit vorhandenen Kooperationsstrukturen in Hinblick auf die heute und in Zukunft zu meisternden gemeinsamen Herausforderungen in der Raumentwicklung weitergedacht werden können. Das Forschungsprojekt "Gemeinsam wachsen, gemeinsam handeln?" widmet sich dieser Frage. Es wurde im Jahr 2018 von der Wiener Wohnbauforschung in Auftrag gegeben und am Institut für Geographie und Regionalforschung der Universität Wien bearbeitet.

Im Rahmen dieses Beitrags sollen zwei der zentralen forschungsleitenden Fragen des Projekts vorgestellt und die Forschungsergebnisse in einen theoretischen Kontext eingebettet werden:

- Inwieweit wird der Intensität der stadtregionalen Verflechtungen in der Stadtregion ${ }^{+}$in Form von interkommunalen Kooperationen derzeit Rechnung getragen?

- Wie lassen sich konkrete Handlungserfordernisse und Handlungsräume innerhalb der Stadtregion+ identifizieren, die (als konzeptioneller Baustein eines strategischen Planungsansatzes), kooperative und projektorientierte Planungen ermöglichen? 
Damit ist in diesem Beitrag die gesamträumliche Ebene der Stadtregion ebenso Gegenstand von Analyse und Diskussion wie stadtregionale Teilräume, die sich durch ähnliche Entwicklungsdynamiken und gemeinsame Planungsherausforderungen auszeichnen. Hauptbestandteile des bewusst breit angelegten Untersuchungsdesigns sind die Analyse funktionaler Verflechtungen, eine akteurbezogene Netzwerkanalyse sowie die Berechnung eines dynamischen Verflechtungsindex, um der komplexen Ausgangslage gerecht zu werden und analytisch fundierte Schlussfolgerungen und Empfehlungen auf unterschiedlichen Maßstabsebenen treffen zu können. Zwei wesentliche Resultate dieser Analysen sind das "Verflechtungs-Kooperations-Paradoxon" und die Leitidee der „flexiblen Regionalisierungen“, die nachfolgend vorgestellt und diskutiert werden. Abschließend lässt sich anhand dieser Ergebnisse weiterführend für die Stadtregion ${ }^{+}$eine kombinierte Strategie als Handlungsempfehlung ableiten: Sie besteht zum einen aus einem integrierten gesamträumlichen Entwicklungsleitbild und zum anderen aus damit verbundenen projektorientierten Umsetzungsmaßnahmen in konkreten stadtregionalen Fokusräumen.

In Kapitel 2 erfolgt zunächst eine theoretische Einbettung des Beitrags in Hinblick auf stadtregionale Kooperationsformen, strategische Planung und soft spaces, um später die Ergebnisse und Empfehlungen unserer Studie entsprechend einordnen zu können. In Kapitel 3 wird die institutionelle Ausgangslage der Stadtregion Wien skizziert und die zentralen Strategiepapiere und Institutionen eingeführt. Nachfolgend werden die beiden zentralen Erkenntnisse der Studie vorgestellt: das Verflechtungs-Kooperations-Paradoxon (Kapitel 4) sowie die Fokusräume und die Leitidee der flexiblen Regionalisierungen (Kapitel 5). In beiden Kapiteln erfolgt dafür zunächst die Erörterung des methodischen Zugangs und im Anschluss die detaillierte Darstellung der Ergebnisse. Im Anschluss findet sich eine kritische Reflexion des methodischen Zugangs (Kapitel 6), bevor abschließend Handlungsempfehlungen präsentiert werden, die einerseits auf die Stadtregion ${ }^{+}$und andererseits auch auf den deutschsprachigen Diskurs im Allgemeinen Bezug nehmen (Kapitel 7).

\section{Theoretische Einbettung}

Stadtregionen rücken in Österreich als räumliche Bezugsebene für kooperative und grenzüberschreitende Aktivität in der integrierten räumlichen Entwicklungsplanung, beispielsweise im Rahmen der natio- nalen „Agenda Stadtregionen“ (ÖROK 2015; Humer 2018: 636 f.), zunehmend in den Fokus. In der österreichischen Gesamtschau zeigen sich unterschiedliche Organisationsformen und Formalisierungsgrade stadtregionaler Zusammenarbeit (Priebs 2019: 73). Der Formalisierungs- und Institutionalisierungsgrad der Bundeshauptstadt Wien und ihres Verflechtungsraumes ist hierbei vergleichsweise gering ausgeprägt. So muss die Situation in der Stadtregion ${ }^{+}$gegenwärtig als "stadtregionales Nebeneinander" eingestuft werden, in dem es keine „stadtregionale Institution für gemeinsame Aufgabenwahrnehmung" gibt (Priebs 2019: 119). Stadtregionale Organisationsformen in Form von Zweckverbänden oder Regionalverbänden, wie sie etwa in deutschen Stadtregionen, z. B. im Ruhrgebiet oder in der Region Hannover, existieren (Zimmermann 2017: 254), finden sich folglich in der Stadtregion Wien nicht. Im Wiener Kontext werden bislang eher Organisationsstrukturen der informellen Kooperation diskutiert. Dies ist unter anderem darauf zurückzuführen, dass sich mit Wien und Niederösterreich im engeren Verflechtungsbereich der „Wiener Stadtregion ${ }^{+4}$ zwei Bundesländer gegenüberstehen, deren hoheitliche Planungskompetenzen an den Ländergrenzen enden.

Aus planungstheoretischer Perspektive liefert die strategische Planung „als integrative und entwicklungsorientierte Form der Planung" (Wiechmann 2019: 7) eine konzeptionelle Grundlage, um informelle Formen stadtregionaler Kooperationen im gesamtstädtischen Kontext zu denken. Die Stärke im Ansatz der strategischen Planung liegt dabei erstens in der Kombination langfristiger (gesamträumlicher) Zielsetzungen mit projektorientierten, (teilräumlichen) Vorgehensweisen (Kühn 2008: 234 ff.). Zweitens ist inre konzeptionelle ,Beweglichkeit‘ in Bezug auf planungsbezogene Ungewissheiten und sich verändernde Rahmenbedingungen hervorzuheben (Weiland/Wohlleber-Feller 2007: 297). Gerade in Großstadtregionen stellen räumliche Veränderungsdynamiken und wechselnde Akteurkonstellationen komplexe strategische Herausforderungen für die Planung dar, auf die eine projektorientierte Vorgehensweise unmittelbarer reagieren kann. Ansätze, die sich lediglich auf formale oder technische Aspekte der räumlichen Planung konzentrieren, sind gerade in nur wenig institutionalisierten Stadtregionen weniger zielführend als entsprechend breiter und flexibler gefasste Ansätze wie die strategische Planung (Hutter/Wiechmann/Krüger 2019: 14).

Als anschlussfähig an die strategische Planung erweist sich in dieser Hinsicht das relationale Konzept der soft spaces. Soft spaces beschreiben Handlungsräume, die sich losgelöst von bestehenden administrati- 
ven Raumabgrenzungen aufgrund funktionaler Verflechtungen, gemeinsamer räumlicher Problemlagen und spezifischer Akteurkonstellationen zu einer bestimmten Zeit ergeben (Allmendinger/Haughton 2009; Sielker/ Chillla 2015: 45). Dabei entwickeln sich diese informellen Kooperationen parallel oder auch im Widerspruch zu administrativ abgegrenzten und formalen Planungsräumen (Purkarthofer 2018: 1008). Gerade auf teilräumlicher Ebene innerhalb einer Stadtregion können soft spaces einen wichtigen konzeptionellen Beitrag leisten, wenn es darum geht, in einem Verflechtungsraum räumlich und zeitlich variierende Planungsherausforderungen zu erkennen und dafür individuelle Lösungen im Sinne strategischer Planung zu entwickeln. Soft spaces dienen damit als theoretisch-konzeptionelle Brille, die es ermöglicht, Konstellationen aus Räumen, Akteuren, Verflechtungen und Problemlagen zu identifizieren (z. B. innerhalb einer Stadtregion), die sich einer gemeinsamen Planungsherausforderung gegenübersehen. Methodische Ansätze zur Identifikation solcher , weichen Planungsräume' sind zentraler Baustein unseres hier diskutierten Forschungskonzepts.

\section{Ausgangslage in der Stadtregion ${ }^{+}$}

An dieser Stelle werden Strategiepapiere und Institutionen vorgestellt, die inhaltlich, strategisch und organisatorisch einen stadtregionalen Bezug aufweisen. Dies ist notwendig, da die abschließenden Handlungsempfehlungen auf dem Bestehenden aufbauen und zu einer Weiterentwicklung der vorhandenen Strukturen beitragen sollen.

\subsection{Stadtregionale Strategiepapiere}

Als eine Reaktion auf die stetige Zunahme von planungs- und entwicklungspolitischen Herausforderungen, die sowohl die Grenzen der Bundesländer als auch der Gemeinden überschreiten, wurde im Jahr 2011 die sogenannte Stadtregion ${ }^{+}$definiert. Damit wurde das Ziel verfolgt, einen zwischen den drei ,betroffenen' Bundesländern abgestimmten räumlichen Bezugsrahmen für stadtregionale Fragestellungen zu schaffen. In Form eines Policy Papers (Planungsgemeinschaft Ost 2011) wurden unter anderem die räumliche Abgrenzung der Stadtregion ${ }^{+}$und die Grundprinzipien der räumlichen Entwicklung vorgestellt, die für sie gelten sollen.
Die Stadtregion ${ }^{+}$setzt sich aus 272 niederösterreichischen bzw. burgenländischen Gemeinden und Wien mit seinen 23 Gemeindebezirken zusammen und ist derzeit Wohnort für mehr als 2,8 Millionen Menschen. Die für sie formulierten Grundprinzipien der räumlichen Entwicklung folgen dem planerischen Leitmotiv der „strukturierten Stadtregion", einem Entwicklungsmodell, das auf punktaxiale Entwicklung und die Herausbildung polyzentrischer Standorträume abzielt (Planungsgemeinschaft Ost 2011: 49).

Bislang sind allerdings weder die Stadtregion ${ }^{+}$als raumplanerische Bezugseinheit noch die in diesem Zusammenhang formulierten raumordnerischen Grundprinzipien in den Planungs- und Handlungsebenen der einzelnen Bundesländer oder einem länderübergreifenden Strategiedokument so verankert, dass der Status über den eines reinen ,Empfehlungsschreibens' hinausgehen würde. Den diesbezüglich bislang weitesten Vorstoß wagt der Wiener Stadtentwicklungsplan STEP 2025 aus dem Jahr 2014 (Stadt Wien 2014a). Zwar hat das Dokument einen grundsätzlich informellen Charakter, wurde aber vom Wiener Gemeinderat beschlossen und entfaltet dadurch eine gewisse politische Bindungswirkung und Orientierungsfunktion. Der STEP 2025 greift die grundlegenden Inhalte der Stadtregion ${ }^{+}$auf und postuliert sie als einen Kooperationsraum, für den "handlungs- und entscheidungsfähige Strukturen für die regionale Kooperation" geschaffen werden sollen (Stadt Wien 2014a: 94). Im Gegensatz zu seinen Vorgängern nimmt der STEP 2025 damit erstmals dezidiert eine ,Agglomerationsperspektive' ein, indem sich das Land Wien zur partnerschaftlichen Zusammenarbeit mit den Nachbargemeinden und den benachbarten Bundesländern bekennt. Einen Schwerpunkt stellt im STEP 2025 insbesondere die langfristige Siedlungs- und Infrastrukturentwicklung der Stadtregion dar, die in partnerschaftlicher Abstimmung, Koordination und Kooperation mit dem Stadtumland erfolgen soll. Dabei soll der regionale Diskurs in „regionalen Kooperationsräumen“, die konkrete gemeinsame Entwicklungsherausforderungen zu bewältigen haben, stattfinden (Stadt Wien 2014a: 95). Damit wird im STEP 2025 auch ein wesentliches Merkmal von strategischer Planung angesprochen, nämlich ein kooperatives und kommunikatives Prozessverständnis, bei dem die Beteiligung verschiedener öffentlicher und privater Akteure hervorgehoben wird (Vallée/Brandt/Fürst et al. 2012: 179). 


\subsection{Stadtregional orientierte Institutionen}

Neben dem Policy Paper zur Stadtregion ${ }^{+}$(Planungsgemeinschaft Ost 2011) und dem STEP 2025 sind es zwei Institutionen, die im Kontext der stadtregionalen und bundesländerübergreifenden Governance anzuführen sind und für deren Aufwertung sich auch der STEP 2025 ausspricht: die Planungsgemeinschaft Ost (PGO) und das Stadt-Umland-Management (SUM). Die Planungsgemeinschaft Ost wurde im Jahr 1978 als eine via Bundesgesetz verankerte Kooperation (sogenanntes §15a-Abkommen) zwischen den drei Bundesländern Wien, Niederösterreich und Burgenland gegründet (Görgl/Gruber 2015: 274 ff.). Im Rahmen dieser gemeinsamen Organisation werden Kriterien für die Grundlagenforschung erstellt sowie Gutachten und Konzepte, in denen Themen von stadtregionaler Relevanz wie Siedlungsentwicklung, Verkehr und Grünraum im Vordergrund stehen. Die Stadtregion ${ }^{+}$ist dabei häufig Bezugsraum für die von der Planungsgemeinschaft Ost beauftragte stadtregionale Grundlagenforschung (Fassmann/Görgl/Helbich 2009; Döringer/Görgl/Huemer 2014; Görgl/Eder/Gruber et al. 2017) sowie für das Monitoring der Siedlungsentwicklung (Eder/Gruber/Görgl et al. 2018).

Das Stadt-Umland-Management wurde im Jahr 2006 von den beiden Bundesländern Wien und Niederösterreich ins Leben gerufen. Es handelt sich dabei um eine informelle, stadtregional agierende Institution. Der Schwerpunkt des Managements liegt auf der Koordination, Vermittlung und Bewusstseinsbildung in den Themenfeldern Siedlungsentwicklung, Verkehr und Freiraumentwicklung (Schaffer/Zuckerstätter 2012). Der kommunikative Aspekt und das Herstellen einer gleichberechtigten Austauschebene für alle beteiligten Akteure in der Stadtregion stehen dabei im Mittelpunkt der Arbeit. Eine zentrale Aufgabe des Stadt-Umland-Managements ist die Organisation der jährlichen Stadtregionkonferenz, auf der verschiedene Akteure die Zukunft der Stadtregion diskutieren. In jüngster Vergangenheit ist zu beobachten, dass das Stadt-Umland-Management auch verstärkt in Organisation und Durchführung bei URBACT-III- und INTERREG-Projekten mit stadtregionalem Bezug eingebunden ist (vgl. Drasdo 2019). Eine inhaltliche oder strategische Abstimmung zwischen der Planungsgemeinschaft Ost und dem Stadt-Umland-Management erfolgt nicht regelmäßig, sondern fallabhängig.

Insgesamt lassen sich Indizien für ein sich wandelndes Planungsverständnis in der Wiener Stadtregion ${ }^{+}$ finden, das die Stadtregion als funktionalen Bezugs- raum anerkennt und strategische Schritte in Richtung eines stadtregionalen Managements setzt. Gleichzeitig sind diese Ansätze aber auch als Ausdruck eines steigenden raumordnungspolitischen Handlungsdruckes zu werten (Zimmermann/Heinelt 2012: 42). Zugleich zeigt sich auch, dass es keine stadtregionale Gesamtstrategie und damit verbundene umsetzungsorientierte Maßnahmen gibt, sondern verschiedene Ansätze und Institutionen vielmehr nebeneinander existieren und dabei inhaltlich und strategisch nicht immer aufeinander abgestimmt sind.

\section{Die Wiener Stadtregion: Ein „Verflechtungs-Kooperations- Paradoxon"?}

Den Ausgangspunkt für unsere Forschung bildete zunächst die Frage, inwieweit den funktionalen Verflechtungen in der Stadtregion ${ }^{+}$in Form von interkommunalen Kooperationen Rechnung getragen wird. Um sich dieser Forschungsfrage zu nähern, wurden zwei methodische Zugänge gewählt. Zum einen wurde eine einfache funktionale Verflechtungsanalyse der stadtregionalen Pendlerströme durchgeführt, zum anderen wurden mittels einer Netzwerkanalyse interkommunale Kooperationsstrukturen in der Stadtregion ${ }^{+}$erhoben. Nachfolgend werden zunächst die methodischen Zugänge und die Ergebnisse der funktionalen Verflechtungsanalyse und der Netzwerkanalyse diskutiert, bevor sie im Anschluss in Form des "Verflechtungs-Kooperations-Paradoxons" gegenübergestellt werden.

\subsection{Funktionale Verflechtungen}

Die Analyse von Pendlerströmen ist ein gut geeigneter Indikator für die Identifikation von funktionalen Verflechtungen zwischen mehreren Gemeinden bzw. administrativen Einheiten (Blotevogel/Schulze 2010: 258). Anhand von Pendlerverflechtungen können stadtregionale Arbeitsmarktbeziehungen abgebildet werden, die eine zentrale funktionale Komponente stadtregionaler Lebens- und Wirtschaftsräume darstellen. So wurden auch in unserer Studie durch die Analyse von Ein- und Auspendlerströmen funktionale Verflechtungsbeziehungen innerhalb der Stadtregion ${ }^{+}$identifiziert, um zunächst grundsätzliche Aussagen über die Verflechtungsintensität und Verflechtungsmuster zwischen der Stadt Wien und den Umlandgemeinden, aber auch zwischen 
den Umlandgemeinden treffen zu können. Für unsere Analyse wurde auf die letztverfügbaren Daten aus der Pendlerstatistik der Statistik Austria aus dem Jahr 2015 zurückgegriffen. ${ }^{1}$

Mittels der funktionalen Verflechtungsanalyse der Erwerbspendlerströme zeigt sich eine für die Wiener Stadtregion ${ }^{+}$charakteristische Struktur (vgl. Abbildung 1). Die Stadt Wien bildet den zentralen Knoten im funktionalen Netzwerk der Stadtregion ${ }^{+}$, der überwiegende Teil der Pendlerbeziehungen aus dem Umland steht mit Wien im Zusammenhang. Zugleich bildet sich eine dem nachgeordnete polyzentrale Struktur heraus, die einerseits von den funktionalen Beziehungen zwischen Wien und den davon weiter entfernten zentralen Orten geprägt ist, andererseits durch starke Austauschbeziehungen zwischen Wien und den Gemeinden, die sich im unmittelbaren ,Ring' um Wien befinden. Hier finden sich auch stark ausgeprägte Verflechtungen zwischen den dortigen Arbeitsplatzzentren im Umland und anderen Umlandgemeinden. Diese Netzwerkstruktur ist für die Stadtregion $^{+}$seit mehr als einem Jahrzehnt prägend; sie hat sich im Laufe der Jahre dahingehend sogar verfestigt (Planungsgemeinschaft Ost 2017: 70). Inklusive aller Gemeinde- und Binnenpendler wurden im Jahr 2015 1,2 Millionen tägliche Arbeitspendler und 355.000 Schulpendler gezählt. Auch wenn mit etwa 936.000 Pendlern der Großteil auf Pendelbewegungen innerhalb Wiens und seiner Bezirke entfällt, zeigt sich in der kartographischen Darstellung die stadtregionale Vernetzung deutlich.

Durch diesen Analyseschritt wird sichtbar, dass die funktionalen Beziehungen zwischen Wien und seinem Umland stark ausgeprägt sind und eine polyzentrale Netzwerkstruktur ergeben, deren zentraler ,Knotenpunkt' Wien ist. Die funktionalen Abhängigkeiten sind dabei aber nicht nur auf Wien zentriert. Viele Umlandgemeinden sind Ziel von Wohnortverlagerungen aus Wien heraus oder haben einen funktionalen Stellenwert als stadtregionale Arbeitsplatzzentren, die Ziel für Erwerbspendler aus Wien sind. Diese erste Analyse liefert Belege für die gegenseitige funktionale Abhängigkeit zwischen Wien und seinem Umland.

1 Hinweis zu den verwendeten Daten im Forschungsprojekt: Ziel war es, jeweils die letztverfügbaren Daten für die Analysen zu verwenden. Aufgrund von unterschiedlichen Datenverfügbarkeiten/ Datenquellen war es allerdings nicht möglich, für alle Analysen exakt dieselben Zeiträume bzw. Jahre heranzuziehen.

\subsection{Netzwerkanalyse der interkommunalen Kooperationen}

Als ,Gegencheck' zu den funktionalen Verflechtungsbeziehungen standen im Rahmen der Studie interkommunale Kooperationen im Fokus des Interesses. Auf der Basis einer Online-Umfrage bei Gemeinden und Wiener Bezirken (September 2018) wurde eine Netzwerkanalyse durchgeführt (vgl. Jansen 2003; Hollstein/Straus 2006), um abzuklären, zwischen welchen Kommunen bereits interkommunale bzw. stadtregionale Kooperationen bestehen. Ziel dieses Analyseschrittes war es, vorhandene Kooperationsbeziehungen in der Stadtregion in Form einer Netzwerkdarstellung aufzubereiten, um Aufschluss über deren räumliche und thematische Ausprägung zu bekommen. Die Umfrage umfasste Fragen nach den Kooperationsbeziehungen zwischen den Gemeinden in unterschiedlichen Themenbereichen. Vertreter der Gemeinden und Bezirke wurden gebeten, jene Gemeinden anzugeben, mit welchen sie in den Bereichen technische Infrastruktur, soziale Infrastruktur, Wirtschaft, Umwelt, Tourismus oder räumliche Planung und Entwicklung zusammenarbeiten. Zudem wurde im Zuge dessen auch die gewählte Form bzw. Körperschaft der Zusammenarbeit (z. B. informell, Gemeindeverband, Verwaltungsgemeinschaft) erhoben. Von den 55 angefragten niederösterreichischen Gemeinden, die gemäß dem Verflechtungsindex (vgl. Kapitel 5.1) eine sehr hohe funktionale Verflechtung aufweisen (mindestens acht Punkte), nahmen 27 Gemeinden an der Online-Umfrage teil. Zudem konnten von den neun Wiener Außen- bzw. Randbezirken zwei Bezirke für die Umfragen gewonnen werden, sodass insgesamt 29 Fragebögen ausgewertet werden konnten. Ausgefüllt wurden die Fragebögen entweder von Bürgermeisterinnen/Bürgermeistern bzw. Bezirksvorstehern, der Stadtamtsdirektion bzw. Amtsleitung oder Mitarbeiterinnen/Mitarbeitern aus sonstigen Verwaltungsbereichen. Die Ergebnisse wurden mittels einer Netzwerkanalyse-Software (Gephi 0.9.2) in Form von ego-zentrierten Netzwerken aufbereitet. Ego-zentrierte Netzwerke beziehen sich auf die Kooperationsbeziehungen zwischen einer teilnehmenden Gemeinde und ihren Kooperationspartnern. Die teilnehmenden und genannten Gemeinden wurden räumlich verortet und ihre Kooperationsbeziehungen visualisiert.

In Hinblick auf die starken Pendlerverflechtungen zwischen Wien und seinem Stadtumland (vgl. Kapitel 4.1) stellt sich die Frage, inwiefern diese stadtregionalen funktionalen Verflechtungen auch einen denkbaren „politischen Kooperationsraum“ (Blotevogel/Schulze 2010: 258) abbilden. Die Ergebnisse der Netzwerkana- 


\section{Stadtregion ${ }^{+}$}

Pendlerverflechtungen 2015 innerhalb der Stadtregion ${ }^{+}$

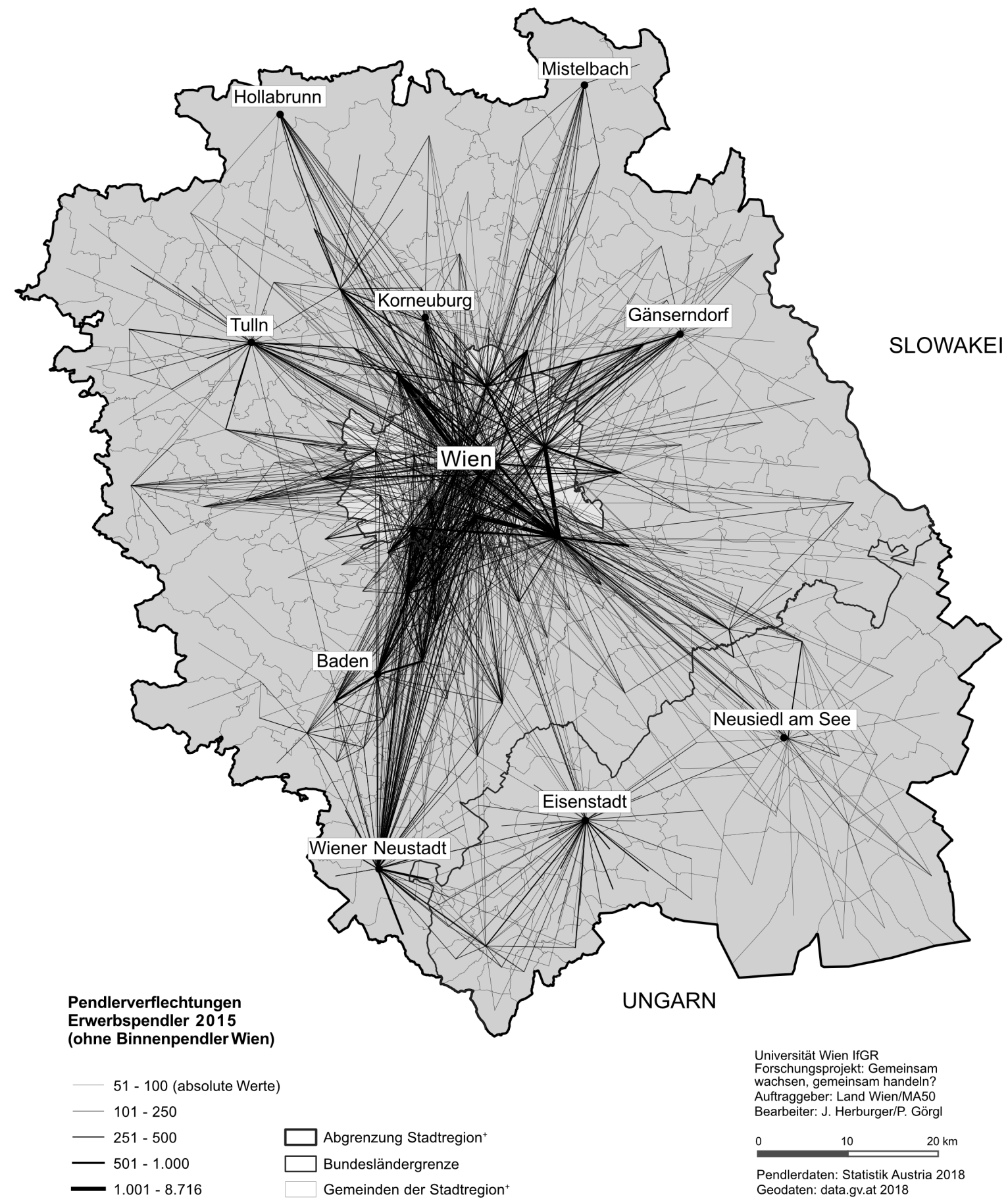

Abbildung 1: Pendlerverflechtungen innerhalb der Stadtregion ${ }^{+}(2015)$ 
lyse widersprechen einem solchen potenziellen Befund. Die im Folgenden näher beschriebenen Kooperationsnetzwerke weisen eine völlig andere Ausrichtung als die funktionalen Verflechtungen auf.

Insgesamt liegen 29 ego-zentrierte Netzwerke vor. Diese Egonetzwerke werden in der Gesamtschau dargestellt, um einen Überblick über die informellen und formellen Kooperationsbeziehungen aller befragten Gemeinden und Bezirke zu bekommen. Die teilnehmenden Gemeinden sind in der Netzwerkdarstellung jeweils rot markiert. Die beiden Wiener Bezirke wurden durch einen Knotenpunkt dargestellt (vgl. Abbildung 2). Für die hier verwendete Darstellung wurden die Antworten der teilnehmenden Gemeinden und Wiener Bezirke unabhängig von den genannten Kooperationsthemen und -formen aggregiert. Das Gesamtnetzwerk umfasst damit 216 Gemeinden (bzw. Knoten) und 1.886 Kooperationsbeziehungen (bzw. Kanten). Angemerkt werden muss, dass aus der Netzwerkdarstellung lediglich Tendenzen in Hinblick auf die Kooperationsstrukturen und das Kooperationsverhalten einzelner Gemeinden abgelesen werden können, da die Stichprobe der befragten Gemeinden relativ gering war. Hinzu kommt, dass sich die Netzwerkdarstellung auf die Perspektive der befragten Gemeinden und Bezirke beschränkt und die genannten kooperierenden (nicht befragten) Gemeinden durchaus weitere Kooperationen aufweisen können.

In den Bezirken Gänserndorf (nordöstliches Umland) sowie Mödling (südliches Umland) ist anhand der Netzwerkstrukturen eine verstärkte Kooperationstätigkeit zwischen den Gemeinden des jeweiligen Bezirks erkennbar. Zudem werden in der Gesamtdarstellung auch die weitreichenden Verflechtungen der nördlichen Umlandgemeinden Richtung Norden, also ,weg von Wien', deutlich. Aus den erhobenen Daten ging zudem hervor, dass die Kooperationen unter den niederösterreichischen Gemeinden vor allem in Form von Zweckverbänden stark ausgeprägt sind. In der Gesamtschau fällt also auf, dass die Stadt Wien eine strukturelle Lücke im Netzwerk darstellt. Insgesamt wird deutlich, dass die Stadt Wien in vergleichsweise geringem Ausmaß in Kooperationsbeziehungen mit den niederösterreichischen Gemeinden steht.

\subsection{Das „Verflechtungs-Kooperations- Paradoxon"}

Anhand der Gegenüberstellung der beiden vorgestellten Analysezugänge lässt sich thesenartig eine zentrale Erkenntnis ableiten: Das interkommunale Koope- rationsnetzwerk verläuft diametral zu den funktionalen Verflechtungen innerhalb der Stadtregion ${ }^{+}$. Wenn es um interkommunale Zusammenarbeit geht, kooperieren die niederösterreichischen Gemeinden im Umland vorwiegend untereinander. Obwohl Wien den Hauptknoten im funktionalen Netzwerk der Stadtregion ${ }^{+}$darstellt, spiegelt sich diese besondere Stellung nicht in Kooperationsbeziehungen wider, sondern wendet sich ins Gegenteil: Wien wurde als Kooperationspartner nur in wenigen Fällen genannt. Diese widersprüchlichen Diagnosen in Bezug auf Verflechtungen einerseits und Kooperationsmuster andererseits werden in Abbildung 3 als das „Verflechtungs-Kooperations-Paradoxon" anschaulich illustriert. Mögliche Erklärungsansätze hierfür sind nicht nur in den unterschiedlichen rechtlichen Rahmenbedingungen, die länderübergreifende Kooperationen erschweren bzw. unmöglich machen, sondern auch in einer bis heute nicht besonders stark ausgeprägten stadtregionalen Planungskultur zu finden (vgl. auch Kapitel 3).

\section{Fokusräume und die Leitidee der flexiblen Regionalisierung}

Aufbauend auf diesen Erkenntnissen stellte sich die Frage nach geeigneten strategischen Planungsansätzen, die das Bewusstsein für eine verstärkte stadtregionale Zusammenarbeit etablieren und eine gemeinsame stadtregionale Planungskultur fördern. Vor dem Hintergrund theoretischer Überlegungen zu soft spaces wurde die Hypothese aufgestellt, dass funktionale Verflechtungen einer räumlichen und zeitlichen Veränderlichkeit unterliegen und damit stadtregionale Kooperationsräume flexibel definiert werden müssen. Zu diesem Zweck wurde ein Verflechtungsindex entwickelt, der die Dynamik und Intensität von funktionalen Verflechtungen in der Wiener Stadtregion ${ }^{+}$widerspiegelt. Dies bildet die Grundlage für die Ableitung von Fokusräumen als kleinräumige, funktionale Teilbereiche innerhalb der Stadtregion+.

\subsection{Der Verflechtungsindex und räumliche Dynamiken in der Stadtregion ${ }^{+}$}

Die räumlichen und zeitlichen Dynamiken von funktionalen Verflechtungen werden in der Fachliteratur anhand verschiedener analytischer Zugänge beleuchtet. So analysiert Rusche (2009) über die Ermittlung einer Wanderungsverflechtungskennziffer 13 Wohnungsmarktregionen, während Kropp und Schwengler (2011) über eine 


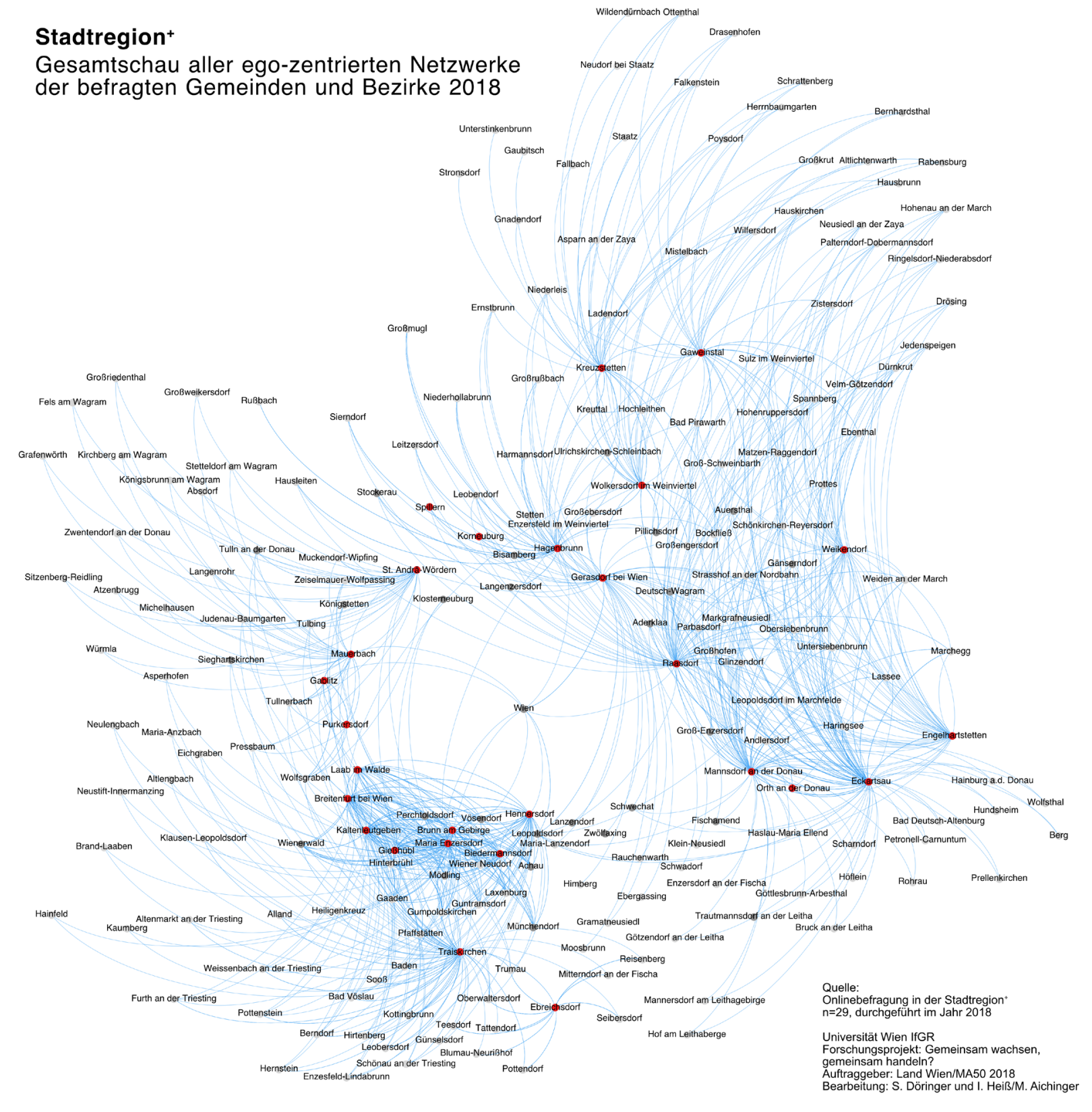

Abbildung 2: Gesamtschau aller ego-zentrierten Netzwerke der befragten Gemeinden und Wiener Gemeindebezirke 2018

graphentheoretische Analyse von Pendlerverflechtungen 50 Arbeitsmarktregionen in Deutschland berechnen. Innerhalb dieser Arbeitsmarktregionen identifizieren sie 50 Kernräume, die über einen Zeitraum von 15 Jahren konstant in der Arbeitsmarktregion lagen. Analysen dieser Art verdeutlichen die Veränderlichkeit von stadtregionalen Verflechtungsräumen.
Um die räumliche Dynamik der funktionalen Verflechtungsbeziehungen in der Stadtregion ${ }^{+}$abbilden zu können, wurde ein Verflechtungsindex, basierend auf Pendler- und Wanderungsdaten für unterschiedliche Zeiträume, entwickelt. Zu Beginn stellte sich die grundsätzliche Frage, wie der Verflechtungsindex berechnet werden soll. Sowohl die Nutzung von Schwellenwerten als auch die Clusteranalyse müssen als methodische 


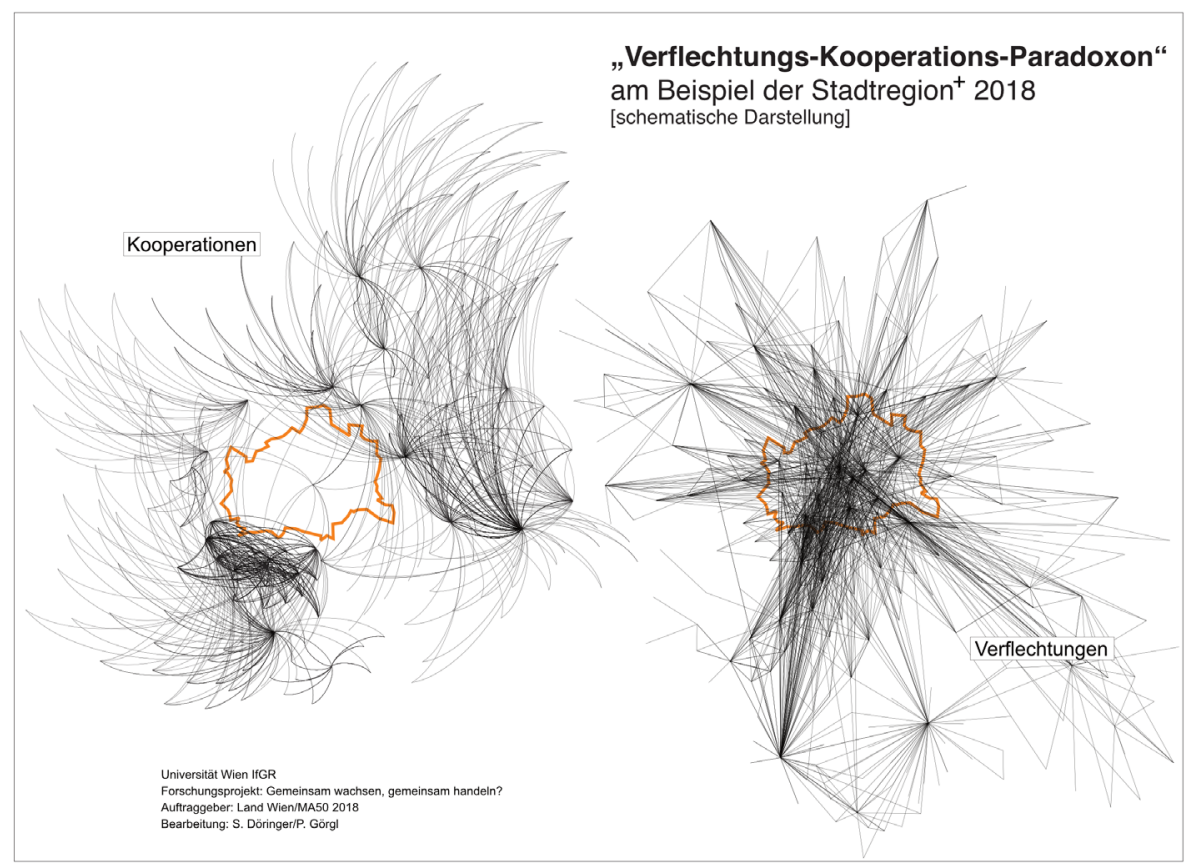

Abbildung 3: Schematische Darstellung des „Verflechtungs-Kooperations-Paradoxons“

Zugänge kritisch reflektiert werden (Kropp/Schwengler 2011: 49). Für die Verwendung von Schwellenwerten, die durch Quartile gebildet werden, spricht, dass es sich um ein transparentes Klassifizierungsverfahren handelt, das sich gut „zur Darstellung von Verflechtungsstrukturen bei Einzugsbereichen" eignet (Bahrenberg/Giese/ Mevenkamp et al. 2010: 49). Die Datenbasis des Verflechtungsindexes bilden je zwei Indikatoren zu Wanderungsverflechtungen und zwei Indikatoren zu Pendlerverflechtungen ${ }^{2}$ (vgl. Tabelle 1).

Je zwei Indikatoren zeigen die Dynamik innerhalb der Gemeinde insgesamt, während wiederum zwei Indikatoren die Verflechtung mit Wien widerspiegeln. Die Analyse erfolgt für zwei Zeiträume; Pendler- und Wanderungsdaten sind aufgrund unterschiedlicher Datengrundlagen dabei nicht für dieselben Perioden verfügbar. ${ }^{3} \mathrm{Da}$

2 Als Datengrundlage für die Berechnung der vier Indikatoren für den Verflechtungsindex dienten uns folgende Datenpakete der Statistischen Datenbank der Statistik Austria (abrufbar über www. statcube.at):

Pendlerdaten: Volkszählung 15.05.2001, Abgestimmte Erwerbsstatistik 31.10.2009 bis 31.10.2015

Bevölkerungsstand: Bevölkerungsregister POPREG 01.01.2002 bis 01.01.2009; 01.01.2013 bis 01.01.2018

Wanderungsdaten: Bevölkerungsregister POPREG 01.01.2002 bis 31.12.2008; 01.01.2013 bis 31.12.2017.

3 Die gewählten Zeiträume wurden gewählt, da sie exemplarisch für die dynamischen Phasen der Bevölkerungsentwicklung Wiens seit der Volkszählung 2001 stehen. im Gegensatz zur eingangs durchgeführten Verflechtungsanalyse (vgl. Kapitel 4.1) beim Verflechtungsindex die Vernetzung der Umlandgemeinden mit Wien im Mittelpunkt des Interesses steht, erfolgte die Analyse monozentriert und ungerichtet: Es fließen also ausschließlich die Verflechtungen der jeweiligen Gemeinde mit der Kernstadt Wien ein, unabhängig von der ,Richtung' der Verflechtung. In Verbindung mit der dynamischen Komponente, die durch die Betrachtung zweier unterschiedlicher Zeiträume gegeben ist, ergeben sich auf diese Weise interessante teilräumliche Befunde über die Verflechtungen der Umlandgemeinden mit Wien.

Die 272 Gemeinden des Wiener Umlands wurden für jeden der vier Indikatoren in Quartile untergliedert, wodurch für jeden Indikator vier gleich große Gruppen entstanden. Je nach Gruppenzugehörigkeit erhielt eine Gemeinde drei, zwei, einen oder keinen Punkt (drei Punkte für Gemeinden des vierten Quartils, also z. B. mit der höchsten Bevölkerungsentwicklung). Die Punktwerte, die die Gemeinden beim jeweiligen Indikator erzielten, wurden schließlich aufsummiert, wodurch eine transparente und gut kommunizierbare Indexzusammenstellung erreicht werden konnte. In den Abbildungen 4 und 5 sind alle mit Wien stark verflochtenen Gemeinden hervorgehoben, die in den jeweiligen Zeiträumen mindestens acht Punkte und damit zwei Drittel der maximalen Punktezahl des Index erreicht haben. Damit eine Gemeinde als stark verflochten eingestuft wird, muss sie 
Tabelle 1: Indikatoren zur Berechnung des Verflechtungsindexes

\begin{tabular}{lcc}
\hline Indikator & Zeitraum 1 & Zeitraum 2 \\
\hline Bevölkerungsentwicklung in \% & $2002-2009$ & $2013-2018$ \\
Anteil der Wanderungen mit Wien an allen Wanderungen der Gemeinde (Zu- und Abwanderungen) & $2002-2009$ \\
in \% & $2013-2018$ \\
Anteil der Pendelbewegungen mit Wien an allen Pendelbewegungen in \% & 2009 \\
Entwicklung der Pendlerzahlen (Ein- und Auspendler) in \% & $2001-2009$ & $2009-2015$ \\
\hline
\end{tabular}

Datengrundlagen: Statistik Austria, Volkszählung 2001, Abgestimmte Erwerbsstatistik 2009 und 2015, POPREG 2002-2009 und 2013-2018 (Bearbeitung: J. Herburger)

also entweder bei allen vier Indikatoren zumindest über dem Median im dritten Quartil liegen (= 4 × 2 Punkte) oder aber bei mindestens zwei Indikatoren im vierten Quartil liegen.

Während im Zeitraum 2002 bis 2009 vor allem Gemeinden im nördlichen Umland intensive Pendlerund Wanderungsverflechtungen mit Wien aufwiesen (vgl. Abbildung 4), haben sich in den Jahren 2009 bis 2018 die Verflechtungen vor allem in den östlichen Gemeinden der Wiener Stadtregion+ intensiviert (vgl. Abbildung 5). Die Verflechtungen mit den westlichen Umlandgemeinden sowie den südöstlichen Umlandgemeinden bleiben über beide Zeiträume hinweg vergleichsweise konstant. In diesem Zusammenhang ist anzumerken, dass die suburban geprägten Gemeinden entlang der Südachse bzw. der Westachse enge und jahrzehntelang gewachsene funktionale und morphologische Verflechtungen mit der Stadt Wien haben. Diese Verbindungen schlagen sich allerdings gegenwärtig nicht mehr in einer besonders dynamischen Entwicklung von Pendler- und Wanderungsströmen nieder, was dazu führt, dass die Gemeinden einen vergleichsweise niedrigen Wert im Verflechtungsindex aufweisen. Dennoch bringen diese Räume Handlungserfordernisse einer ,gereiften Suburbia' mit sich. Dafür seien exemplarisch die Bewältigung des Individualverkehrs, die Abstimmung des öffentlichen Nahverkehrs oder die Freihaltung von regionalen Grünräumen genannt (Stadt Wien 2005: 150 ff.).

Worin liegt der Mehrwert des Verflechtungsindex in Anbetracht dessen, dass sich Entwicklungsherausforderungen auch in weniger stark verflochtenen Gemeinden aufgrund der allgemein herrschenden Dynamik ergeben? Mithilfe des Verflechtungsindexes lässt sich zunächst aufzeigen, in welchen Teilbereichen der Stadtregion $^{+}$diese Entwicklungsdynamik derzeit gerade besonders stark mit der Kernstadt Wien in Zusammenhang steht. Damit verbunden sind ähnliche Herausforderungen in verschiedenen Policy- und Planungsfeldern (z. B. Umgang mit erhöhter Baulandnachfrage, steigender Pendlerverkehr, Bedarf an P+R-Plätzen, erhöhte Konkurrenz um Flächen). Dabei handelt es sich großteils um solche Aufgabenfelder, die von vielen Gemeinden, ,traditionell' immer noch als genuin kommunal wahrgenommen werden. Mithilfe des Verflechtungsindexes kann aber aufgezeigt werden, wo es zusammenhängende Teilbereiche innerhalb der Stadtregion gibt, die eine gemeinsame ,Betroffenheit' aufweisen und wo demnach auch überkommunal planerischer Handlungsund Abstimmungsbedarf besteht. Solchermaßen identifizierte Teilräume werden im Projekt als „Fokusräume“ bezeichnet. Sie stellen soft spaces dar, für die jeweils geeignete Governance-Modelle diskutiert und ausgearbeitet werden können. Ein wesentliches Charakteristikum ist dabei die Relationalität der Verflechtung zwischen Wien und den Fokusräumen. Die von der Stadt Wien ausgehenden Dynamiken können sich demnach auch in Teilbereichen der Stadtregion niederschlagen, die räumlich nicht unmittelbar an die Bundeshauptstadt angrenzen. Ebenso sei angemerkt, dass Fokusräume an sich keine starre Raumeinheit darstellen sollen, sondern der räumliche Umgriff, wiederum im Sinne des Softspaces-Konzepts, erweiterbar ist, wenn dies inhaltlich, projektbezogen oder strategisch notwendig sein sollte. Was das in der ,Umsetzung 'bedeutet, wird im folgenden Kapitel anhand eines der drei identifizierten Fokusräume exemplarisch gezeigt.

\subsection{Stadtregionale Fokusräume}

\subsubsection{Exemplarische Auswahl des Fokusraums „Wiener Norden und nördliches Wiener Umland“}

Im Rahmen der Studie wurde anhand eines Fokusraums exemplarisch diskutiert, welche vertiefenden Analyseschritte auf dieser Ebene anwendbar sein können 


\section{Stadtregion $^{+}$}

Verflechtungsindex 2002-2009

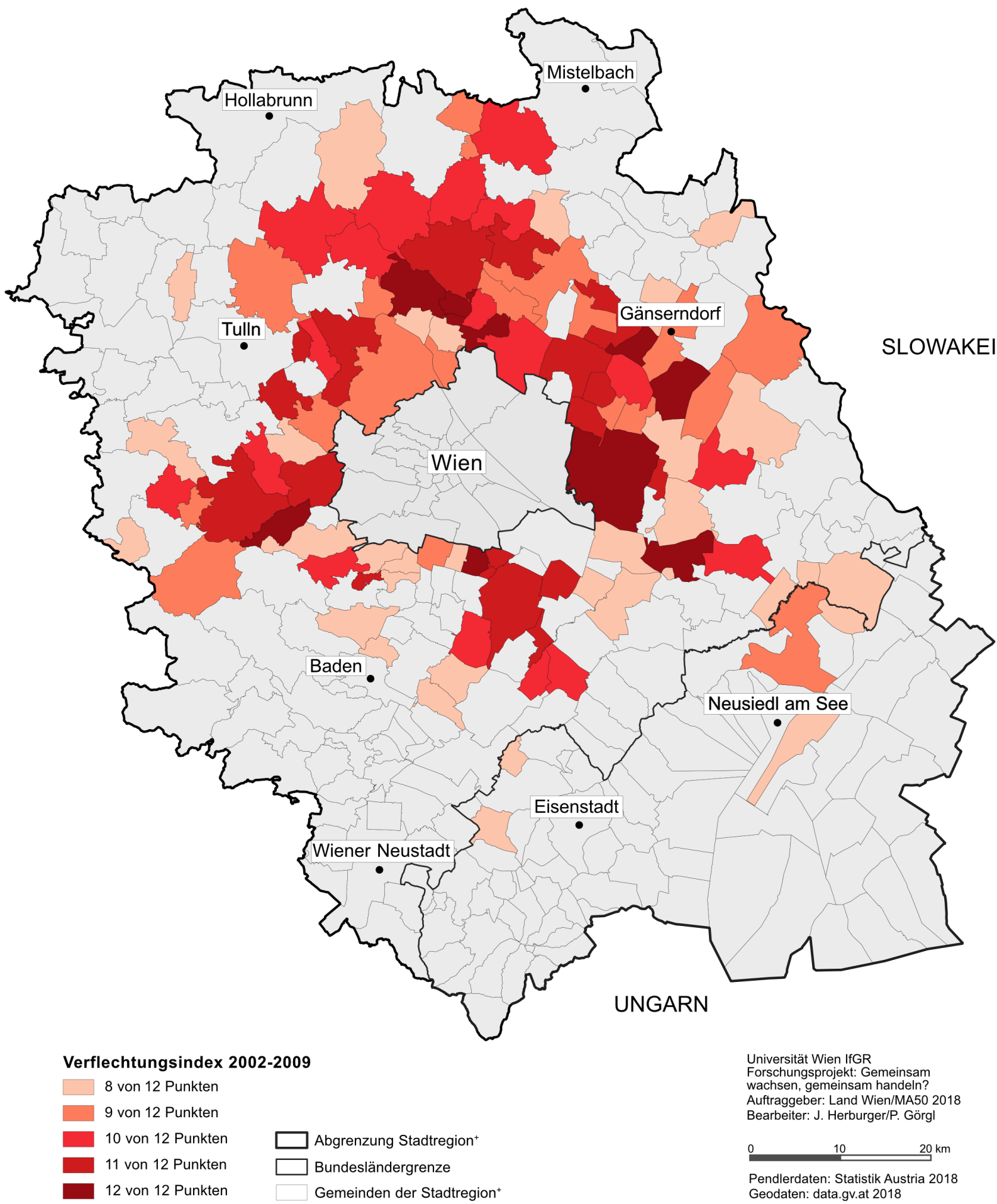




\section{Stadtregion ${ }^{+}$}

Verflechtungsindex 2009-2018

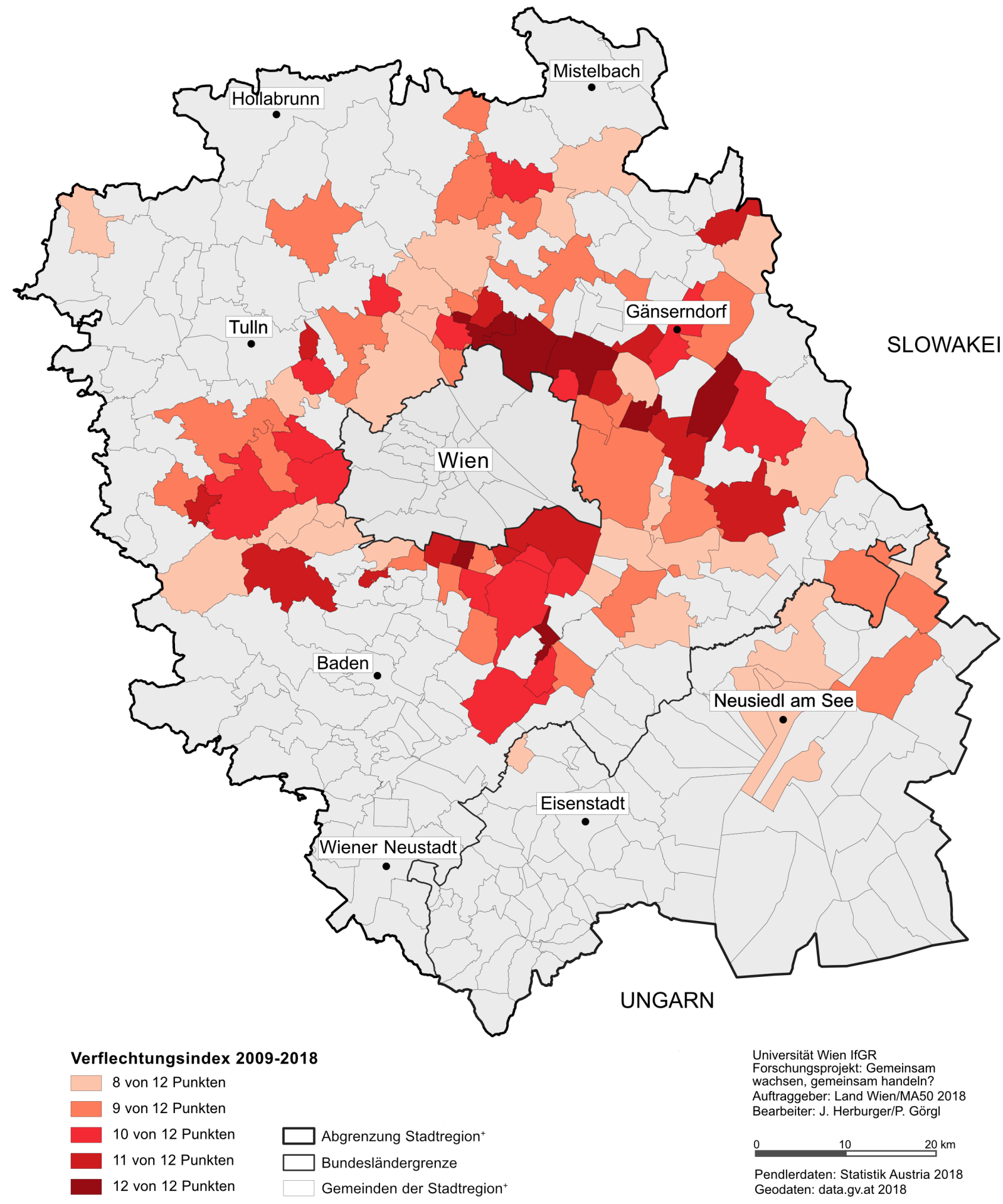

Abbildung 5: Verflechtungsindex für den Zeitraum 2009-2018 
und wie sie als Grundlage für darauf aufbauende, teilraumspezifische Planungsschritte eingesetzt werden könnten. Näher betrachtet wurde von uns der Fokusraum „Wiener Norden und nördliches Wiener Umland“, der aus sechs zum Teil unmittelbar an Wien angrenzenden Umlandgemeinden besteht. Durch seine direkte Nachbarschaft zu Wien erschien es zunächst wichtig, den ursprünglich mittels Verflechtungsindex identifizierten Fokusraum um den 21. Wiener Gemeindebezirk zu erweitern. Es handelt sich dabei, so die Hypothese, insgesamt um einen Teilraum, der trotz seiner unterschiedlichen räumlich-strukturellen Voraussetzungen gemeinsame Planungs- und Entwicklungsherausforderungen zu bewältigen hat. Anhand dieses Fokusraumes werden zwei Aspekte diskutiert: Es soll aufgezeigt werden, welche raumanalytischen Zugänge auf dieser Betrachtungsebene gewinnbringend anwendbar sein können und worin ihr Mehrwert im Kontext eines interkommunalkooperativen Planungsansatzes liegen kann.

\subsubsection{Analyse des Fokusraums „Wiener Norden und nördliches Wiener Umland"}

Die sechs mittels Verflechtungsindex identifizierten niederösterreichischen Umlandgemeinden des Fokusraums zählten im Jahr 2018 zusammen knapp 41.000 Einwohner. Sie grenzen zum Teil direkt an den 21. Wiener Gemeindebezirk Floridsdorf an (Bevölkerungsstand im Jahr 2018: 163.000). Dies zeigt auf den ersten Blick die unterschiedlichen Größenverhältnisse zwischen der Bundeshauptstadt und ihrem Umland und die damit verbundenen Schwierigkeiten, wenn eine räumlich-statistische Analyse die Gemeindeebene als räumliche Bezugseinheit verwendet. Die Aussagekraft bleibt entsprechend oberflächlich. Deshalb wurde auf rasterbasierte Daten zurückgegriffen, die nicht an administrative Grenzen gebunden sind. Diese analytische Ebene bringt verschiedene Vorteile mit sich. Zum einen sind in statistischen Rastern mit 250 m Kantenlänge sehr kleinräumige Aussagen zu planungsrelevanten Merkmalsausprägungen möglich (z. B. Bevölkerung, Haushalte, Gebäude und Wohnungen, Arbeitsstätten). Zum anderen treten auf Rasterebene eventuell vorhandene, ,grenzüberschreitende' räumlich-strukturelle Zusammenhänge deutlicher zutage (Wonka/Kaminger/ Katzlberg 2009: 662 f.). Dies bringt gerade in Hinblick auf strategische und kooperativ ausgerichtete Planungsansätze einen deutlichen Mehrwert.

Abbildung 6 stellt die absolute und die relative Bevölkerungsentwicklung zwischen 2011 und 2018 im Fokusraum kombiniert dar. Wenig überraschend weist Wien-Floridsdorf die höchsten Absolutwerte auf. Betrachtet man die Entwicklung hingegen in der Relation, so zeigt sich, dass in einigen Rasterzellen der Umlandgemeinden ein im Verhältnis ebenso starkes bzw. sogar stärkeres Wachstum stattgefunden hat. Das eingangs angesprochene "Wachstumsmanagement" ist also ganz offensichtlich im Umland ebenso zu leisten wie in der Großstadt. Eine diesbezügliche Analyse kann dadurch nicht nur ,grenzüberschreitend' und kleinräumig solche gemeinsamen Dynamiken identifizieren, sondern dadurch auch eine inhaltliche Grundlage für interkommunal-kooperative Planungsdialoge und Strategiefindungsprozesse darstellen.

Die Abbildungen 7 und 8 zeigen die Entwicklung der Wohngebäude mit einer bis zwei Wohneinheiten bzw. mit drei oder mehr Wohneinheiten. Hier wird zunächst deutlich, dass der Einfamilienhausbau nach wie vor klar im Trend liegt und es sich dabei nicht um ein ausschließliches Umlandphänomen handelt. Auch in WienFloridsdorf werden in einigen Rasterzellen sehr hohe Werte erreicht. Bei der Analyse der Entwicklung von Wohngebäuden mit mehr als drei Wohneinheiten zeigt sich hingegen der deutliche räumliche Fokus auf Wien und die Bezirksstadt Korneuburg. Eine diesbezügliche ,Urbanisierung des Umlands' lässt sich damit nicht nachweisen. Auffällig ist aber die im Vergleich zum Einfamilienhausbau geringe Anzahl an Rasterzellen, in denen im betrachteten Zeitraum dichtere, mehrgeschossige Wohnformen realisiert wurden. Gerade in sich schnell entwickelnden suburbanen Teilräumen ist beispielsweise die Diskussion über „Verdichtung“ (bzw. „zu viel Verdichtung") allgegenwärtig. Kleinräumige Analysen dieser Art können eine Faktengrundlage liefern, auf der entsprechend fundiert diskutiert werden kann. Ebenso könnte diese Analyse wertvolle Beiträge liefern, wenn es etwa um die Identifizierung von und den planerischstrategischen Umgang mit Siedlungs- und Entwicklungshotspots in einem Teilraum geht.

In Hinblick auf die Etablierung der teilraumbezogenen Raumentwicklung im Rahmen einer strategischen Gesamtplanung sind sowohl Fokusräume als Bezugseinheit als auch vertiefende, kleinräumige Analysen ein aus unserer Sicht vielversprechender konzeptioneller Zugang.

\subsection{Flexible Regionalisierungen}

Hinsichtlich der räumlichen und zeitlichen Dynamik der funktionalen Verflechtungen und den sich daraus erge- 
Fokusraum Wiener Norden und nördliches Umland Bevölkerungsentwicklung 2011-2018

Absolut

- $-159--50$

- $-49--10$

$-9-0$

$0-10$

- $11-50$

- $51-100$

- $101-250$

C) $251-500$

$501-1.134$

in Prozent

$-100 \%--25 \%$

$-25 \%--10 \%$

$-10 \%-<0 \%$

$>0 \%-10 \%$

$>0 \%-10 \%$

$25 \%-500 \%$

$25 \%-230 \%$
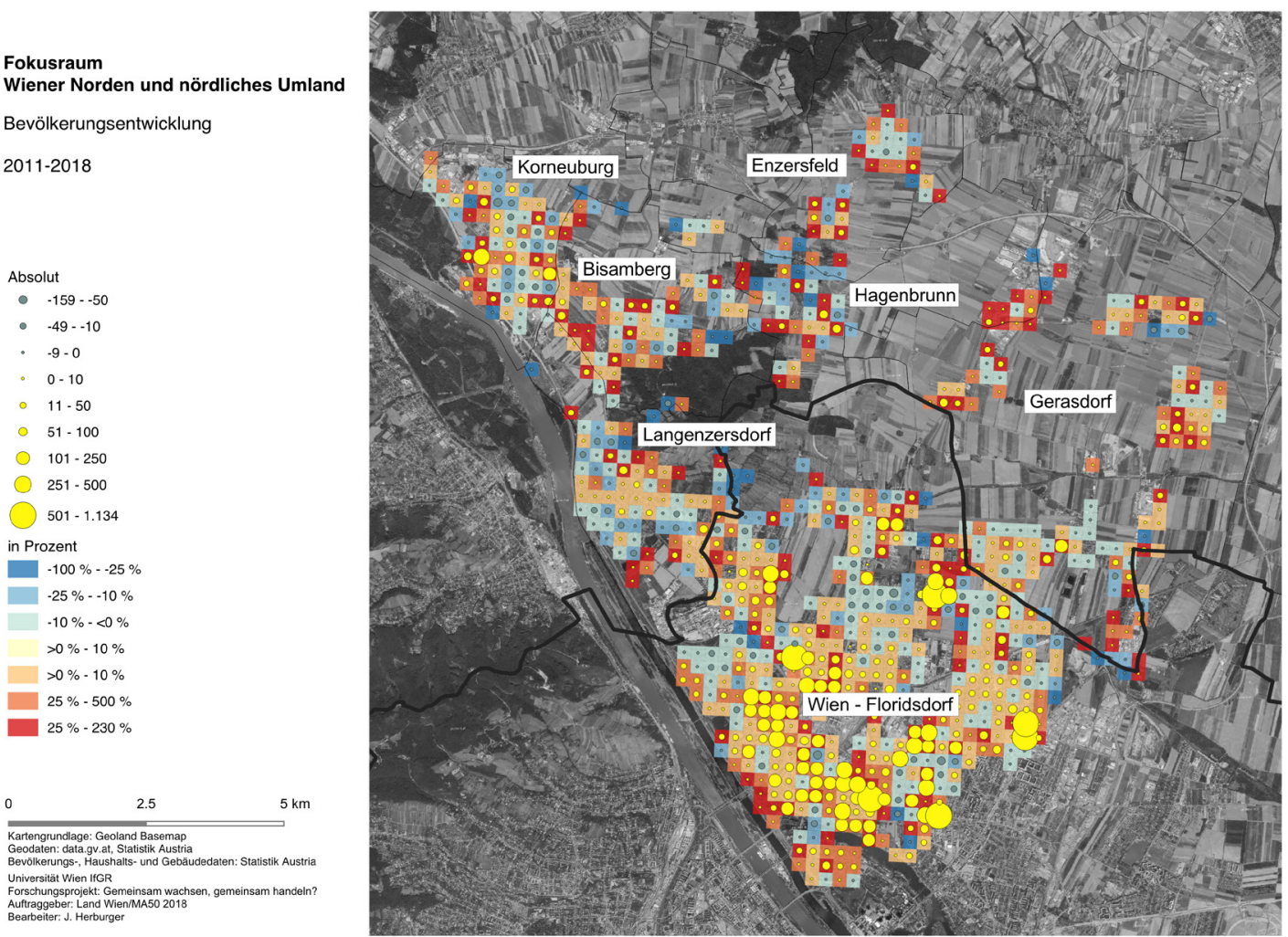

Abbildung 6: Bevölkerungsentwicklung 2011-2018 im Fokusraum „Wiener Norden und nördliches Umland“
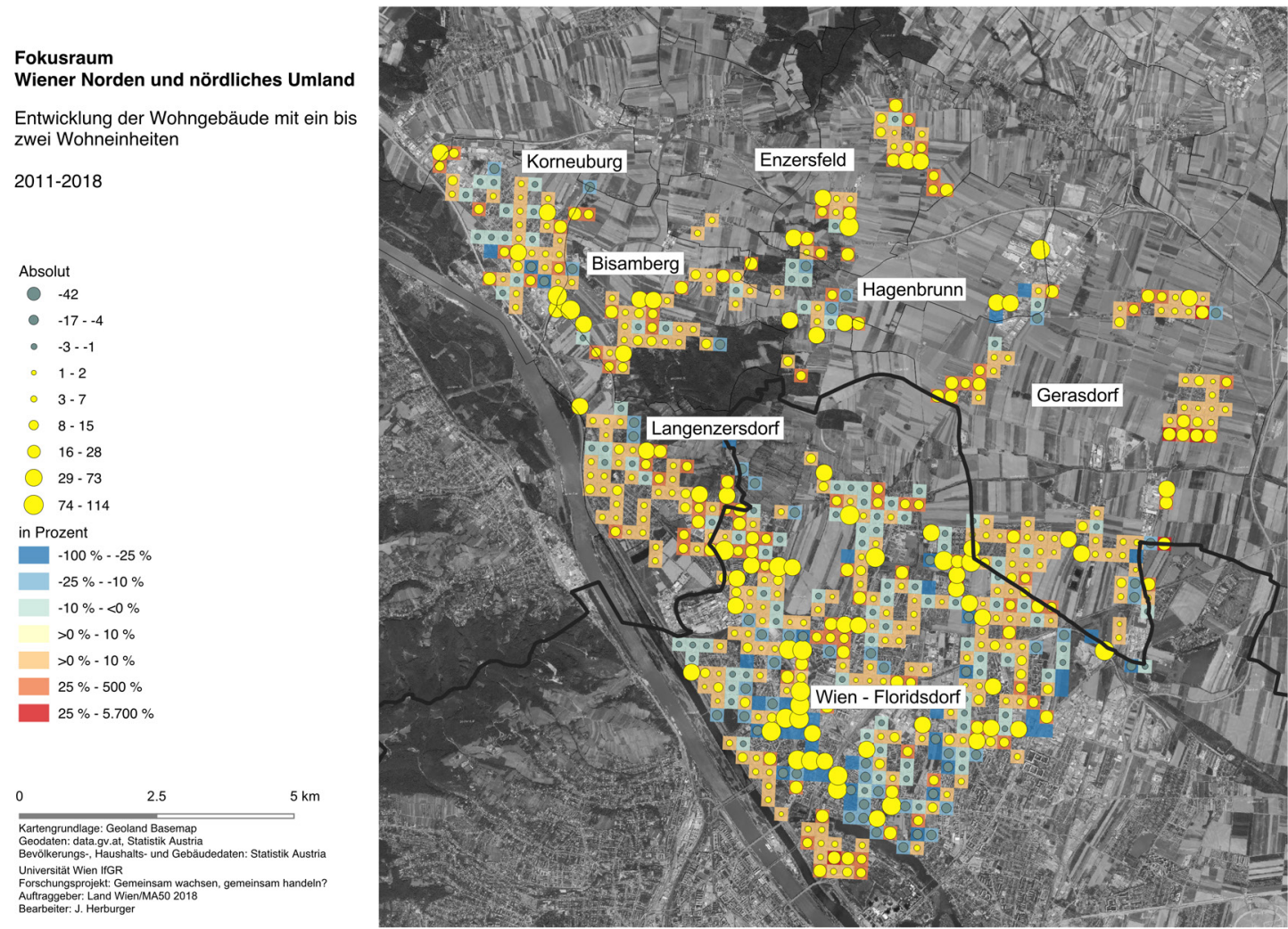

Abbildung 7: Entwicklung der Wohngebäude mit ein bis zwei Wohneinheiten 2011-2018 im Fokusraum „Wiener Norden und nördliches Umland" 

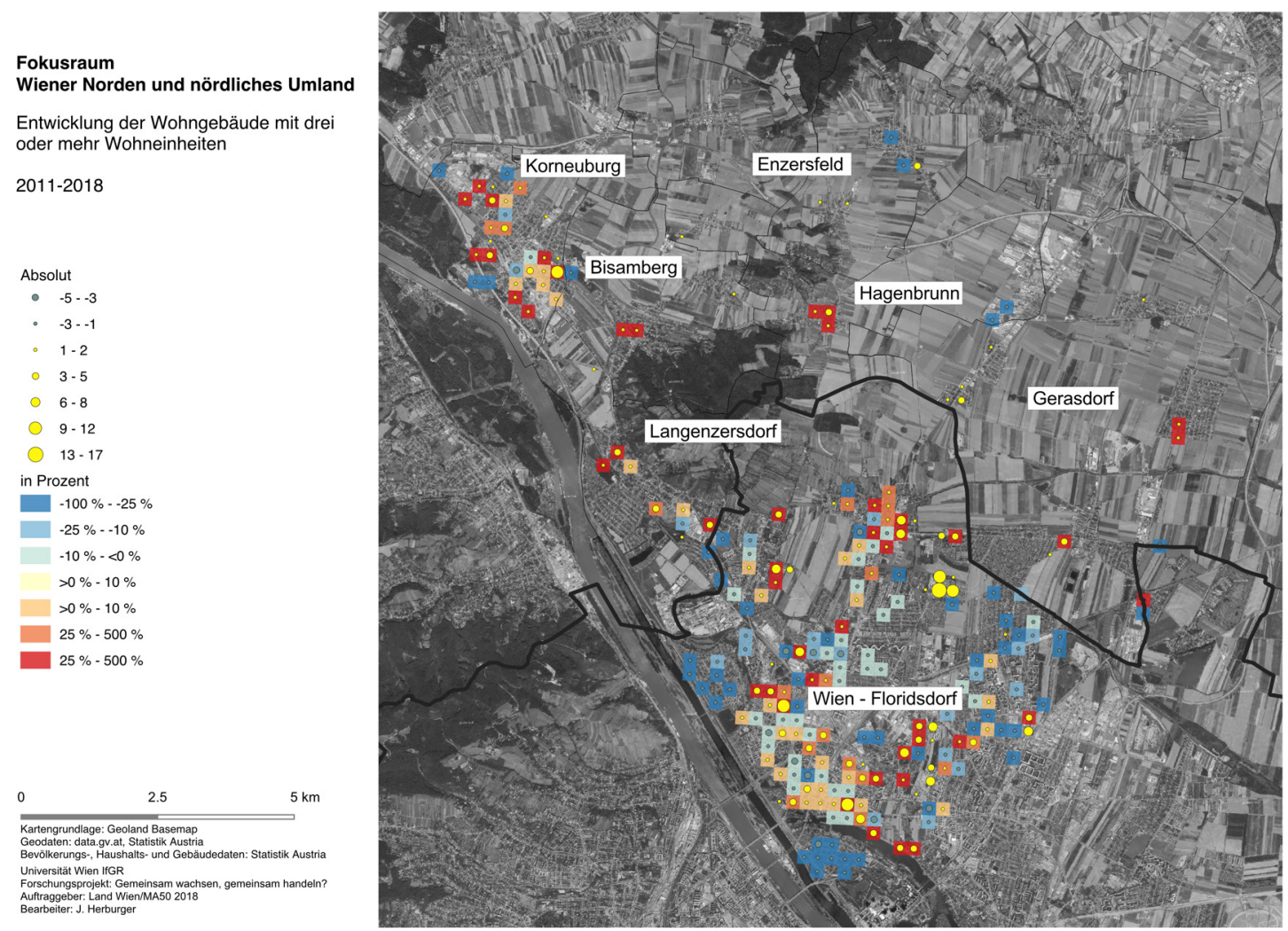

Abbildung 8: Entwicklung der Wohngebäude mit drei und mehr Wohneinheiten 2011-2018 im Fokusraum „Wiener Norden und nördliches Umland“

benden Handlungs- und Kooperationserfordernissen plädieren wir für die Leitidee der flexiblen Regionalisierungen. Mit dem Ansatz der flexiblen Regionalisierungen und der eingeführten räumlichen Bezugsebene der Fokusräume möchten wir ein Augenmerk auf räumliche Teilausschnitte in Stadtregionen legen, die flexibel und problembezogen definiert werden können. Der Verflechtungsindex als Analyseinstrument und flexible Regionalisierungen als ,planerisches Grundprinzip könnten demnach im Wiener Beispiel dafür herangezogen werden, die im STEP 2025 geforderten und eingangs erwähnten „regionalen Kooperationsräume“" (Stadt Wien 2014a: 95) zu implementieren. Neben der Weiterentwicklung des strategischen Planungsverständnisses im stadtregionalen Kontext kann man dabei an das relationale Konzept der soft spaces (vgl. Kapitel 2) anknüpfen. Die Vorzüge von ,weichen Räumen', die jenseits von administrativen Grenzen und formell verankerten Planungsinstrumenten definiert werden, liegen dabei insbesondere in der raschen und flexiblen Problemlösungskompetenz und in der anpassungsfähigen Organisation der Entscheidungsstrukturen (vgl. Allmendinger/Haughton 2009; Purkarthofer/Humer 2019).

\section{Methodenreflexion und Diskussion}

Um Aussagen über den Status Quo von Kooperationsstrukturen in einer Stadtregion treffen zu können, hat sich die Netzwerkanalyse als ein probater Zugang erwiesen. Trotz der geringen Fallzahlen in dieser Studie konnten klare Kooperationsmuster und Kooperationsintensitäten zwischen einzelnen Städten und Gemeinden in der Stadtregion für unterschiedliche Themenbereiche der interkommunalen Zusammenarbeit herausgearbeitet werden. Dieser Zugang könnte auch in bestehenden, stärker institutionalisierten Stadtregionen interessant sein (Breidenbach/Kambeck/Matz et al. 2013: 148 ff.), um beispielsweise im Zuge eines Monitorings Kooperationsstrukturen abzufragen und abzubilden. Hierfür ist es wichtig, entsprechend hohe Responseraten erzielen zu können bzw. eine Vollerhebung unter allen Gemeinden einer Stadtregion anzustreben. Auf diese Weise wäre auch die Berechnung von quantitativen Maßzahlen und Kennwerten für die Netzwerke möglich, die eine tiefergehende quantitative Analyse der Netzwerkstrukturen zulässt (Jansen 2003: 127 ff.). 
Dass die Kombination verschiedener analytischer Zugänge zu interessanten Ergebnissen führen kann, zeigt sich am "Verflechtungs-Kooperations-Paradoxon" (vgl. Kapitel 4.3). Durch den an sich simplen Vorgang der Gegenüberstellung der Ergebnisse wird pointiert deutlich, wie gegensätzlich sich im Fall der untersuchten Stadtregion ${ }^{+}$funktionale Vernetzungen und stadtregionale Kooperationsstrukturen abbilden. In Vorträgen und öffentlichen Diskussionen erzielte das „VerflechtungsKooperations-Paradoxon" vor allem bei Akteuren aus Planung und Politik eine entsprechende Aufmerksamkeit und führte zu angeregten Diskussionen darüber, ob und wie die stadtregionale Kooperation im Wiener Kontext intensiviert werden könnte.

Der in der Studie entwickelte Verflechtungsindex ist eine nützliche räumlich-statistische Hilfsgröße und bietet eine Alternative zu klassischen funktionalen Verflechtungsanalysen, wie z. B. Pendlerverflechtungen (Blotevogel/Schulze 2010: $262 \mathrm{ff}$.). Ziel der Indexbildung ist es, miteinander zusammenhängende Teilbereiche innerhalb einer Stadtregion zu identifizieren, die gegenwärtig stärkere Verflechtungen mit der Kernstadt aufweisen als andere Räume der Stadtregion. Damit können spezifische Planungs- und Entwicklungsherausforderungen verbunden sein (Stichwort Wachstumsmanagement), auf die die stadtregionale Planung entsprechend eingehen kann. Insgesamt konnte gezeigt werden, dass die Verflechtungsintensitäten im Zeitverlauf wechseln und zu unterschiedlichen Zeitpunkten jeweils andere Teilbereiche stärker mit der Kernstadt verflochten sind. Im Kontext der Stadtregion ${ }^{+}$lag das Erkenntnisinteresse dezidiert auf den Verflechtungen des Umlands mit der Kernstadt Wien, entsprechend wurde die Bundeshauptstadt als Zentrum und statistische Bezugsgröße definiert. Welche Ergebnisse die Berechnung eines Verflechtungsindexes in stark ausgeprägten polyzentralen Stadtregionen ohne klar dominierende Kernstadt (vgl. Growe/Heider/Lamker et al. 2012) liefert, müsste in einer weiterführenden Untersuchung geklärt werden. Aufbauend auf den Analyseergebnissen unserer Studie konnten damit sowohl das Prinzip der flexiblen Regionalisierungen als auch die Planungsebene der Fokusräume abgeleitet werden, welche als neuartige konzeptionelle Bausteine Eingang in strategisch orientierte, stadtregionale Planungsansätze und Leitbilder finden können.

Die Idee der Fokusräume könnte sich im Fall der Stadtregion $^{+}$als ein in mehrfacher Hinsicht vielversprechender konzeptioneller Zugang erweisen. Im Sinne eines strategischen Planungsansatzes sind Fokusräume als Planungs-, Umsetzungs- und Kooperationsebene gleichermaßen relevant. Vor allem können sie einen wesentlichen Beitrag dazu leisten, dass sich kleinere Umlandgemeinden und die aus deren Sicht oftmals als „übergroß“ empfundene Kernstadt auf Augenhöhe begegnen (Zimmermann 2018: 1215). Die vorgestellte kleinräumige, rasterbasierte Analyse leistet viel, um zu zeigen, wo die Umlandgemeinden dieselben Ausgangsbedingungen und möglicherweise auch dieselben Planungsherausforderungen zu meistern haben wie der Stadtbezirk in ihrer unmittelbaren Nachbarschaft. So können Fokusräume als weiche Planungsräume bzw. soft spaces definiert werden, in denen jeweils projektorientiert, kooperativ und grenzüberschreitend gearbeitet wird. Ihre konzeptionelle Stärke liegt darin, dass auf diese Weise die übergeordneten Planungs- und Entwicklungsziele eines strategischen Gesamtleitbilds teilräumlich ,individualisiert' werden und zugleich Lösungen für spezifische planerische Herausforderungen für Teilbereiche der Stadtregion entwickelt werden können.

\section{Handlungsempfehlungen}

In der Großstadtregion Wien sind wechselseitige Kooperationsstrukturen derzeit nur sehr schwach ausgeprägt. Zwar sind mit der „Wiener Stadtregion"“ und Strategiepapieren wie dem Wiener Stadtentwicklungsplan STEP 2025 inhaltlich-konzeptionelle Ansätze für eine strategisch ausgerichtete Raumentwicklung gegeben. Ebenso gibt es mit der Planungsgemeinschaft Ost und dem Stadt-Umland-Management zwei Institutionen, die bundesländerübergreifend agieren und fallweise auch in die projektbezogene Zusammenarbeit zwischen der Stadt Wien und den Umlandgemeinden eingebunden sind (vgl. Kapitel 3.2). De facto ist die Stadt-Umland-Kooperation derzeit aber auf ein Mindestmaß beschränkt, vor allem, wenn man den vergleichsweise hohen Institutionalisierungs- und ,Reifegrad' deutscher Stadtregionen bedenkt (vgl. Zimmermann/Heinelt 2012; Breidenbach/ Kambeck/Matz et al. 2013). Das in Kapitel 4.3 beschriebene "Verflechtungs-Kooperations-Paradoxon" kann in diesem Kontext als sinnbildlich für diesen Status Quo verstanden werden. In der Weiterführung der im Beitrag diskutierten Ergebnisse sehen wir die besten Chancen für die Weiterentwicklung stadtregionaler Kooperationsstrukturen in der Implementierung von informell-kooperativen Raumentwicklungsansätzen. Damit können im Sinne eines strategischen Planungsansatzes ein übergeordnetes, integriertes räumliches Leitbild als Orientierungsrahmen für die gesamte Stadtregion ${ }^{+}$angestrebt werden und zugleich umsetzungsbezogene Strategien für spezifische Teilräume entwickelt werden. Mit dieser 
Vorgehensweise kann auf dem Bestehenden Schritt für Schritt aufgebaut werden und sich eine neue Form von stadtregionsbezogenem Problembewusstsein bzw. eine stadtregional orientierte Planungskultur entwickeln.

Um dieses Ziel zu erreichen, sind allerdings verschiedene Hürden für eine kooperative Raumentwicklung aus dem Weg zu räumen, seien sie institutioneller Natur, durch bestimmte Akteurkonstellationen oder durch widerständige Verhaltensmuster bei (potenziellen) Kooperationspartnern bestimmt (Knieling 2003: 471). Dies trifft nicht nur auf den speziellen Wiener Fall zu, sondern lässt sich durchaus auf andere Stadtregionen übertragen. Die in unserem Forschungsprojekt diskutierten Analysemethoden und planerischen Leitideen können dazu beitragen, einige dieser Hürden zu überwinden, wenn man sie als Elemente einer strategischen Planung versteht, in der die kommunikative und diskursive Dimension im Vordergrund steht (Knieling 2006: 478). Dies gilt für kooperativ angelegte Raumentwicklungsprozesse allgemein und speziell dort, wo zuerst ,Basisarbeit' geleistet werden muss, weil das Bewusstsein für die (Stadt-)Region bei den relevanten Akteuren nur schwach oder gar nicht vorhanden ist. In diesem Zusammenhang können analytische Zugänge wie der vorgestellte Verflechtungsindex (vgl. Kapitel 5.1) oder das erwähnte „VerflechtungsKooperations-Paradoxon" einen diskursiven Beitrag zur ,regionalen Bewusstseinsbildung' leisten. Damit lassen sich interkommunale Zusammenhänge prägnant aufzeigen und damit verbundene Planungsherausforderungen faktenbezogen diskutieren.

Ebenso übertragbar scheinen die aus der Betrachtung der Wiener Stadtregion ${ }^{+}$abgeleiteten Fokusräume und die Leitidee der flexiblen Regionalisierungen (vgl. Kapitel 5.2 und 5.3). Strukturelle Flexibilität ist ein zentrales Merkmal strategischer Planung (Kühn 2008: 236). In unserem Verständnis betrifft dies die räumliche Dimension ebenso wie die zeitliche; hier setzen die flexiblen Regionalisierungen als ,Planungsprinzip‘ an. Unter dem strategischen Dach eines integrierten räumlichen Leitbilds rücken bewusst kontinuierlich neue Teilräume für eine bestimmte Zeit in den Fokus der Raumentwicklung. Innerhalb dieser Teilräume werden in interkommunalen Fokusräumen mit allen jeweils relevanten Akteuren gemeinsame Planungs- und Entwicklungsherausforderungen identifiziert und kooperativ an deren Lösung und Umsetzung gearbeitet. Auf dieser Ebene steht ein „pragmatisch-integratives Aufgabenverständnis“ im Vordergrund (Knieling 2003: 467) und es bietet sich die Chance, dass sich verschiedene Akteure auf Augenhöhe begegnen. Solche ,Planungsräume' innerhalb einer Stadtregion sollten im Sinne flexibler Regionali- sierungen also nichts Statisches sein, sondern bewusst ,weiche Räume' (inhaltlich, akteurbezogen und auch deren Abgrenzung betreffend). Die Raumentwicklung in einer Stadtregion kann davon insgesamt profitieren, da der konzeptionelle Mehrwert in der räumlichen, inhaltlichen und situativen Flexibilität liegt. Darüber hinaus kann ein stark akteurbezogen-kooperativer Zugang dieser Art dazu beitragen, dass sich die Identifikation mit Planungsentscheidungen oder räumlichen Leitbildern ebenso erhöht wie das Bewusstsein für eine regionale Perspektive bei Fragen der räumlichen Entwicklung. Ein mit diesem Zugang zur räumlichen Entwicklungsplanung einhergehender kollektiver Lernprozess (Danielzyk/Sondermann 2018: 971) könnte so am Ende die titelgebende Frage des Forschungsprojekts "Gemeinsam wachsen, gemeinsam handeln?" in ein Motto für stadtregionale Raumentwicklung umwandeln: Gemeinsam wachsen, gemeinsam handeln!

Danksagung: Wir danken dem Auftraggeber, dem Referat Wohnbauforschung und internationale Beziehungen (MA 50) der Stadt Wien, für die gute Zusammenarbeit und die wertvollen Diskussionen während der Projektlaufzeit.

\section{Literatur}

Allmendinger, P.; Haughton, G. (2009): Soft spaces, fuzzy boundaries, and metagovernance: the new spatial planning in the Thames Gateway. In: Environment and Planning A: Economy and Space 41, 3, 617-633. doi: 10.1068/a40208

Bahrenberg, G.; Giese, E.; Mevenkamp, N.; Nipper, J. (2010): Statistische Methoden in der Geographie. Band 1: Univariate und bivariate Statistik. Stuttgart.

Blotevogel, H. H.; Schulze, K. (2010): 1 oder 2 oder 3? Zur Konstituierung möglicher Metropolregionen an Rhein und Ruhr. In: Raumforschung und Raumordnung 68, 4, 255-270. doi: 10.1007/s13147-010-0040-8

Breidenbach, P.; Kambeck, R.; Matz, F.; Schmidt, C. M. (2013): Jeder für sich oder doch mehr für alle? Wege zu einer intensiveren regionalen Kooperation im Ruhrgebiet. In: Raumforschung und Raumordnung 71, 2, 143-156. doi: 10.1007/s13147-0130219-x

Danielzyk, R.; Sondermann, M. (2018): Informelle Planung. In: ARL - Akademie für Raumforschung und Landesplanung (Hrsg.): Handwörterbuch der Stadt- und Raumentwicklung. Hannover, 963-974.

Döringer, S.; Görgl, P.; Huemer, J. (2014): Standort- und Verdichtungspotenziale im Nahbereich von Bahnhöfen und Haltestellen in der Stadtregion plus. Wien.

Drasdo, F. H. (2019): LOS_DAMA! Compendium. Green infrastructure for better living. München. 
Eder, J.; Gruber, E.; Görgl, P.; Hemetsberger, M. (2018): Wie Wien wächst: Monitoring aktueller Trends hinsichtlich Bevölkerungsund Siedlungsentwicklung in der Stadtregion Wien. In: Raumforschung und Raumordnung | Spatial Research and Planning 76, 4, 327-343. doi: 10.1007/s13147-018-0546-z

Fassmann, H.; Görgl, P.; Helbich, M. (2009): Atlas der wachsenden Stadtregion. Materialband zum Modul I des Projekts „Strategien zur räumlichen Entwicklung der Ostregion (SRO)“. Wien.

Görgl, P.; Eder, J.; Gruber, E.; Fassmann, H. (2017): Monitoring der Siedlungsentwicklung in der Stadtregion+. Strategien zur räumlichen Entwicklung der Ostregion. Wien.

Görgl, P.; Gruber, E. (2015): Die kooperative Stadt der Zukunft? Stadt-Umland-Zusammenarbeit am Beispiel der Stadtregion Wien. In: Fritz, J.; Tomaschek, N. (Hrsg.): Die Stadt der Zukunft. Aktuelle Trends und zukünftige Herausforderungen. Münster, 267-280.

Growe, A.; Heider, K.; Lamker, C.; Paßlick, S.; Terfrüchte, T. (Hrsg.) (2012): Polyzentrale Stadtregionen - Die Region als planerischer Handlungsraum. Hannover. = Arbeitsberichte der ARL 3.

Hanika, A.; Fuchs, R.; Klotz, J. (2015): ÖROK-Regionalprognosen 2014-2030. Teil 1: Bevölkerung. Wien. = ÖROK-Schriftenreihe 196/1.

Hollstein, B.; Straus, F. (2006): Qualitative Netzwerkanalyse: Konzepte, Methoden, Anwendungen. Wiesbaden. doi: 10.1007/978-3-531-90074-2

Humer, A. (2018): Linking polycentricity concepts to periphery: implications for an integrative Austrian strategic spatial planning practice. In: European Planning Studies 26, 4, 635652. doi: $10.1080 / 09654313.2017 .1403570$

Hutter, G.; Wiechmann, T.; Krüger, T. (2019): Strategische Planung. In: Wiechmann, T. (Hrsg.): ARL Reader Planungstheorie. Band 2: Strategische Planung - Planungskultur. Berlin, 13-25. doi: 10.1007/978-3-662-57624-3

Jansen, D. (2003): Einführung in die Netzwerkanalyse: Grundlagen, Methoden, Forschungsbeispiele. Wiesbaden. doi: 10.1007/9783-663-09875-1

Knieling, J. (2003): Kooperative Regionalplanung und Regional Governance: Praxisbeispiele, Theoriebezüge und Perspektiven. In: Informationen zur Raumentwicklung 8/9, 463-478.

Knieling, J. (2006): Leitbilder und strategische Raumentwicklung. Planungstheoretische Einordnung und Diskussion der neuen Leitbilder für die deutsche Raumentwicklung. In: Raumforschung und Raumordnung 64, 6, 473-485. doi: 10.1007/BF03183113

Kropp, P.; Schwengler, B. (2011): Abgrenzung von Arbeitsmarktregionen - ein Methodenvorschlag. In: Raumforschung und Raumordnung 69, 1, 45-62. doi: 10.1007/ s13147-011-0076-4

Kühn, M. (2008): Strategische Stadt- und Regionalplanung. In: Raumforschung und Raumordnung 66, 3, 230-243. doi: 10.1007/BF03183159

ÖROK - Österreichische Raumordnungskonferenz (2015): Für eine österreichische Stadtregionspolitik. Agenda Stadtregionen in Österreich. Empfehlungen der ÖREK-Partnerschaft „Kooperationsplattform Stadtregion“. Wien.

Planungsgemeinschaft Ost (2011): Stadtregion ${ }^{+}$. Planungskooperation zur räumlichen Entwicklung der Stadtregion Wien, Niederösterreich, Burgenland. Zwischenbericht. Wien.

Planungsgemeinschaft Ost (2017): Monitoring der Siedlungsentwicklung in der Stadtregion+. Wien.

Priebs, A. (2019): Die Stadtregion: Planung - Politik - Management. Stuttgart.

Purkarthofer, E. (2018): Diminishing borders and conflating spaces: A storyline to promote soft planning scales. In: European Planning Studies 26, 5, 1008-1027. doi: 10.1080/09654313.2018.1430750

Purkarthofer, E.; Humer, A. (2019): City-regional policies in the planning systems of Finland and Austria: National initiatives and European opportunities. In: Belgeo 2. doi: 10.4000/ belgeo.32122

Rusche, K. (2009): Abgrenzung von Wohnungsmarktregionen mithilfe von Wanderungsverflechtungen: eine vergleichende Fallstudie. In: Raumforschung und Raumordnung 67, 1, 34-44. doi: 10.1007/BF03183141

Schaffer, H.; Zuckerstätter, R. (2012): SUM: Informal cooperation in the Vienna metropolitan region. In: Regions Magazine 285, 1 , 14-15. doi: 10.1080/13673882.2012.10515097

Sielker, F.; Chilla, T. (2015): Regionen als ,Soft Spaces‘? Das neue EU-Instrument der makroregionalen Strategien. In: Kühne, O.; Weber, F. (Hrsg.): Bausteine der Regionalentwicklung. Wiesbaden, 41-54. doi: 10.1007/978-3-658-02881-7_4

Stadt Wien (2005): STEP 05 - Stadtentwicklung Wien 2005. Wien. Stadt Wien (2014a): STEP 2025 - Stadtentwicklungsplan - Mut zur Stadt. Wien.

Stadt Wien (2014b): Wien wächst ... Bevölkerungsentwicklung in Wien und den 23 Gemeinde- und 250 Zählbezirken. Wien. = Statistik-Journal Wien 1/2014

Vallée, D.; Brandt, T.; Fürst, D.; Konze, H.; Priebs, A.; Schmidt, P. I.; Scholich, D.; Tönnies, G. (2012): Modell einer Strategischen Regionalplanung in Deutschland. In: Vallée, D. (Hrsg.): Strategische Regionalplanung. Hannover, 170-190. = Forschungs- und Sitzungsberichte der ARL 237.

Weiland, U.; Wohlleber-Feller, S. (2007): Einführung in die Raumund Umweltplanung. Paderborn.

Wiechmann, T. (2019): Einleitung - Zum Stand der deutschsprachigen Planungstheorie. In: Wiechmann, T. (Hrsg.): ARL Reader Planungstheorie. Band 2: Strategische Planung - Planungskultur. Berlin, 1-11. doi: 10.1007/978-3-66257624-3

Wonka, E.; Kaminger, I.; Katzlberg, G. (2009): Regionalstatistische Auswertungen mit geographischen Rastern in der Raumplanung. In: Informationen zur Raumentwicklung 10/11, 661-675.

Zimmermann, K. (2017): Re-Scaling of Metropolitan Governance in Germany. In: Raumforschung und Raumordnung | Spatial Research and Planning 75, 3, 253-263. doi: 10.1007/s13147017-0480-5

Zimmermann, K. (2018): Kooperation, informelle und regionale. In: ARL - Akademie für Raumforschung und Landesplanung (Hrsg.): Handwörterbuch der Stadt- und Raumentwicklung. Hannover, 1211-1220.

Zimmermann, K.; Heinelt, H. (2012): Metropolitan Governance in Deutschland: Regieren in Ballungsräumen und neue Formen politischer Steuerung. Wiesbaden. doi: 10.1007/978-3-53119159-1 